\title{
Mechanics of mystery
}

\author{
Peter Nick $^{1}$
}

Received: 30 January 2017 / Accepted: 30 January 2017 / Published online: 6 February 2017

(C) Springer-Verlag Wien 2017

Machines as models for living organisms have been central for the development of modern biology, and when we try to understand a biological phenomenon, we strive for "mechanistic insight". Thus, the science of machines, mechanics, has turned into a synonym for "real explanation". In an ironic twist of science history, this enthusiasm for machine-based explanations for living phenomena contrasts with a still relatively poor understanding of the way, how mechanic forces actually shape biology or how cells can perceive them. This is certainly not due to a minor role of mechanics for biology; in contrast, many central sensory inputs to signaling are actually manifestations of mechanic force, including such important environmental factors as gravity, or shear force, but also less explicitly mechanic stimuli such as sound, cold, or osmotic challenge. On the side of biological responses, the entire architecture of organisms (as in plants) or the entire organisation of their movements (as in animals) is shaped in response to mechanic inputs. Nevertheless, contemporary methodology is clearly chemistry dominated - we tend to talk about molecules first, less frequently about forces. This represents a real gap in modern biology, a kind of blind spot, because a better understanding of mechanic forces would also allow to get deeper insight into shapes as central topic in cell and developmental biology.

One important reason for this constraint may be the difficulty to administer and measure force on a cellular scale, due

Handling Editor: Reimer Stick

Peter Nick

peter.nick@kit.edu

1 Botanical Institute, Karlsruhe Institute of Technology, Fritz-Haber-Weg 4, Karlsruhe, Germany to technical constraints. Therefore, experimental approaches suffer from the change they inflict to the system they want to measure. This can inspire controversial and never-ending debates on phenomena that even after decades of research remain mysterious and therefore are discussed with often religious zeal. The mechanics underlying these mysteries may be simply of methodological nature. Minimal invasiveness of technology is here the key for new insights as illustrated by two contributions to the current issue.

As a contribution to the new methods in Cell Biology section, Branco et al. (2017) in the current issue use a nanoindentation approach to apply very localized forces to epidermal plant cells and to follow the local response of subcortical actin. The motivation for this type of work is to understand the local defence to fungal penetration attempts, where invading fungi form local appressoria and build up often considerable hydraulic pressures to penetrate the cell wall. As a very general and efficient countermeasure to such penetration attempts, plant cells transport vesicles with cell wall material (often building blocks for callose) to the site to tighten it up by callose papillae. This requires a rapid reorganisation of the actin cytoskeleton in response to the local change of wall mechanics. To get "mechanistic" insight into this mechanical sensing, the forces and the stimulation times are relevant, because they define the dose of the stimulus as very important parameters of any possible working model for the process. Using GFP fusions with the actin-binding domain 2 of plant fimbrin, a widely used marker for life-cell imaging of plant actin, the authors show that actin responds very swiftly - in less than 2 min - and they are able to measure the threshold forces require to elicit such responses to be as low as $4 \mu \mathrm{N}$ over around $20 \mathrm{~s}$. The forces determined for invading fungal or oomycete pathogens are in the range of one order of magnitude higher, which speaks for an extremely efficient sensor. This sensitivity is even more impressive, considering that it 
has to occur on the background of considerable turgor pressure against the cell wall.

Also, the second contribution in the current issue, by former board member, Friedrich Bentrup (2017), deals with the impact of measuring technology on the concepts used to explain a biological phenomenon. The issue is very fundamental - the question, how water can ascent in large trees. There is little controversy about transpiration as central driving force, but there has been a quite controversial debate about the tissues that are involved and about the relevance of concurrent mechanisms. This debate has been mainly shaped by the methodology used to measure the water potential within a tree. While the classical cohesion-tension theory ascribes water transport exclusively to continuous columns of water in the xylem, alternative views invoke also additional mechanisms from the surrounding parenchymatic tissue, such as inverse transpiration, water transport through membrane channels, and water acquisition by gels consisting of pectic wall components. The historical note not only gives an overview on more than a century of controversy around the cohesion theory but also critically reviews the methodology used by the different opponents in this debate. To measure water potential in a reliable manner is far from trivial: central support for the cohesion-tension theory comes from measurements with the Scholander bomb. The interpretation of the readouts obtained by this approach depends strongly on certain models on the mechanic and hydraulic situation within a tree. Again, it was the development of non-invasive techniques, such as nuclear magnetic resonance (Rokitta et al. 1999) that required a new viewpoint. This historical viewpoint on a still ongoing debate nicely demonstrates the conclusion by this author that "new techniques generate new insights".

\section{Compliance with ethical standards}

Conflict of interest The author declares that there is no conflict of interest.

\section{References}

Bentrup FW (2017) Water ascent in trees and lianas: the cohesion-tension theory revisited in the wake of Otto Renner. Protoplasma, current issue

Branco R, Pearsall EJ, Rundle C, White R, Bradby J, Hardham AR (2017) Quantifying the plant actin cytoskeleton response to applied pressure using nanoindentation. Protoplasma, current issue

Rokitta M, Peuke AD, Zimmermann U, Haase A (1999) Dynamic studies of phloem and xylem flow in fully differentiated plants by fast nuclear-magnetic resonance microimaging. Protoplasma 209:126131 